\title{
La place des sciences sociales et humaines dans le système supérieur algérien
}

The place of human and social sciences in Algerian higher education

El lugar de los las ciencias humanas y sociales en el sistema superior argelino

Yassine Mohammed Ferfera et Tchirine Mekideche

\section{CpenEdition}

\section{Journals}

Édition électronique

URL : http://journals.openedition.org/ries/569

DOI : 10.4000/ries.569

ISSN : 2261-4265

Éditeur

Centre international d'études pédagogiques

Édition imprimée

Date de publication : 1 décembre 2008

Pagination : 95-105

ISSN : $1254-4590$

Référence électronique

Yassine Mohammed Ferfera et Tchirine Mekideche, «La place des sciences sociales et humaines dans le système supérieur algérien », Revue internationale d'éducation de Sèvres [En ligne],

49 | décembre 2008, mis en ligne le 01 décembre 2011, consulté le 19 avril 2019. URL : http:// journals.openedition.org/ries/569; DOI : 10.4000/ries.569 


\section{La place des sciences sociales et humaines dans le système supérieur algérien}

\section{Yassine Ferfera Tchirine Mekideche}

Le système d'enseignement supérieur algérien est relativement jeune et résulte d'un processus de réforme entamé dès le recouvrement de la souveraineté nationale. Jusqu'à l'indépendance, la formation supérieure de type universitaire était orientée vers la formation d'une élite coloniale française. Le processus de construction d'un système éducatif national proprement algérien débute avec la création du ministère de l'éducation en 1963. L'université d'Alger, dont la création remonte à $1909^{1}$, constituera le noyau du développement du système d'enseignement supérieur algérien, qui va connaître une croissance quasi exponentielle. Ne dépassant guère 2500 à la rentrée universitaire de septembre 1962, le nombre d'étudiants inscrits pour une formation de graduation a atteint les 800000 étudiants à la rentrée universitaire de 2006/2007. Il dépassera certainement le million à la rentrée de 2008/2009.

Totalement concentrés dans une seule université en 1962, ces effectifs sont désormais répartis dans plus de cinquante établissements qui couvrent tout le territoire national avec une densité appréciable, ce qui répond au principe du service public de proximité. En matière d'offre de formation, la palette complète de disciplines est proposée. Cependant la répartition de cette offre de formation se caractérise encore par de forts déséquilibres dont la correction constitue l'un des principaux objectifs assignés aux efforts déployés par les pouvoirs publics.

Le développement rapide de l'Université algérienne, encore en cours, pose aujourd'hui un double problème : celui de la consolidation de tous ses segments et celui de la correction de ses dysfonctionnements. Il est plus que probable que les difficultés d'ordre organisationnel et fonctionnel des établissements d'enseignement supérieur sont en partie liées au rythme exceptionnellement accéléré de leur croissance.

1. L’École de médecine et de pharmacie fut créée à Alger en 1859. En 1879 s'ajoutèrent la faculté des sciences, celle de lettres puis de droit. En 1909, ces facultés sont regroupées avec d'autres instituts, pour donner naissance à l'Université d'Alger, l'une des plus anciennes d'Afrique. 


\section{LES QUATRE ÉTAPES \\ DU DÉVELOPPEMENT DU SYSTÈME D'ENSEIGNEMENT SUPÉRIEUR}

L'évolution de l'institution universitaire algérienne dans ses structures et curricula a connu quatre étapes essentielles depuis l'indépendance. La première a consisté dans la prise en charge de l'héritage colonial. La deuxième correspond à la mise en œuvre de la réforme du système d'enseignement supérieur de 1971. La troisième est celle du développement extensif, avec la mise en place de la carte universitaire de 1982 - actualisée en 1984 - et de l'arabisation de l'enseignement. Enfin, la période récente qui démarre avec la promulgation de la loi n 99-05 du 04-04-99 sur l'enseignement supérieur est marquée par la volonté de trouver une nouvelle rationalité au système en conformité avec les bouleversements que connaît le pays.

À l'indépendance, l'Algérie hérite d'une université organisée en facultés disciplinaires académiques, caractérisée par un cloisonnement extrême et à vocation élitiste. Issu du modèle français, ce système d'enseignement supérieur avait une orientation plus académique que professionnelle. L'enseignement à caractère technologique, qui n'avait pas droit de cité à l'Université, était organisé dans des établissements spécialisés. L'organisation de l'Université accordait peu d'autonomie à la faculté qui était plus une structure à caractère pédagogique et scientifique qu'administrative. La faculté regroupait un ensemble de disciplines caractérisées par un certain degré de proximité, bien qu'assez autonomes les unes par rapport aux autres, par exemple le droit et les sciences économiques, les mathématiques et la physique, la biologie et la chimie, la sociologie et l'histoire, etc. Parallèlement à cela, sur le plan spatial, la carte universitaire était réduite à sa plus simple expression. L'essentiel des établissements d'enseignement supérieur était regroupé dans la capitale.

Il n'est pas exagéré de dire que la véritable université algérienne est apparue avec la réforme de l'enseignement supérieur de 1971. Les exigences du modèle de développement économique, dont la mise en œuvre a commencé à partir de 1967, imposaient une mutation du système d'éducation et de formation en général et de l'enseignement supérieur en particulier. En effet, le schéma d'industrialisation conçu par l'État induisait des besoins importants en cadres supérieurs qui ne pouvaient alors être satisfaits. Une nouvelle orientation et de nouvelles missions sont définies et assignées à l'Université. La réforme, qui vise une intensification de la croissance de l'enseignement supérieur, instaure une refonte totale des programmes de formation (avec un objectif de développement de l'enseignement de la technologie dans les différents cursus) et de l'organisation des études.

De surcroît, on lance une réorganisation totale des structures universitaires dont la principale caractéristique consiste dans la substitution des instituts d'université spécialisées aux facultés. 
C'est ainsi que la faculté des sciences a éclaté en plusieurs instituts, chacun d'eux centré sur un domaine scientifique : instituts de mathématique, de physique, de chimie, d'informatique, de biologie, des sciences de la terre. La faculté de droit et des sciences économiques s'est scindée en instituts des sciences juridiques et administratives, de sciences politiques, des sciences économiques. La faculté des lettres et sciences humaines s'est scindée en instituts de psychologie et sciences de l'éducation, de sociologie, de philosophie, d'histoire, de lettres, Toutefois, cette spécialisation des instituts n'a pas empêché certains regroupements de se faire. Ce mode d'organisation s'inspirait largement du modèle des universités anglo-saxonnes où les sciences appliquées sont développées autour d'un solide socle constitué par les sciences fondamentales.

Peu de temps après la réforme de 1971, on vit apparaître de nombreux décalages par rapport aux objectifs définis initialement. On observait en particulier que le libre accès à l'enseignement supérieur ne mettait pas un terme au déséquilibre dans la répartition des effectifs entre les différentes filières. La situation s'aggrava même avec l'accroissement des bacheliers au cours de cette période. Par ailleurs, les nouvelles modalités d'organisation des cursus s'avérèrent trop complexes et, sur le plan administratif, l'autonomie des instituts ne se concrétisa pas réellement.

C'est pourquoi, dès le début des années quatre-vingts, un certain nombre de mesures vont être mises en ouvre afin d'assurer une meilleure adéquation entre la formation supérieure et les besoins de l'économie nationale. Parmi ces mesures à caractère essentiellement correctif, on mentionnera l'intégration explicite de l'enseignement supérieur dans le processus global de planification nationale. Pour la première fois, des objectifs quantitatifs précis de formation de cadres par branches et secteurs d'activité sont assignés à l'enseignement supérieur. Cela revêt la forme de la carte universitaire de 1982 (réactualisée en 1984) qui traduit les besoins en termes de production annuelle de diplômés par discipline et filière de formation. On note aussi une tentative de mieux réguler les flux des différentes filières, en fixant des conditions restrictives d'accès, de manière à éviter l'engorgement de certains curricula.

À l'issue de cette évolution extrêmement rapide, la situation de l'Université algérienne se caractérisait, à la rentrée universitaire 1997-1998, par un ensemble de dysfonctionnements problématiques. En premier lieu, on pouvait noter le gonflement considérable des effectifs dans certaines filières avec de sérieuses difficultés de prise en charge se traduisant par une efficacité et un rendement des plus faibles. De fait, la gestion des effectifs n'obéissait plus aux normes minimales souhaitables : normes d'accueil, normes d'encadrement, etc. On constatait également la tendance à l'expansion, par effet de «clonage», de la carte universitaire en dehors des normes exigibles, notamment en matière d'encadrement pédagogique ${ }^{2}$.

2. Les troubles politiques de la période poussèrent de surcroît de nombreux universitaires au départ vers d'autres pays, ce qui renforça le problème d'encadrement. 
Par ailleurs, la cohabitation de treize universités, treize centres universitaires, dix instituts nationaux qui sont, d'une manière générale, monodisciplinaires, neuf ENS/ENSET mono-disciplinaires, dix grandes écoles et instituts nationaux spécialisés, posait problème. Loin d'être une source de richesse, cette variété constituait un élément essentiel du processus de génération des dysfonctionnements. En effet, l'extrême diversification organisationnelle était à l'origine de la parcellisation des missions et des taches, du cloisonnement des disciplines et des comportements, de la réplication des formations, de l'isolement et du repli sur soi des enseignants et des chercheurs, de la limitation des complémentarités et des synergies, de la sous-optimalité dans l'utilisation des ressources et des moyens humains, matériels et financiers, de l'absence de toute flexibilité de fonctionnement du système.

Il devint clair qu'il fallait concevoir un dispositif unificateur ayant pour objectif majeur la rationalisation du système dans son ensemble. Ce fut l'un des objectifs recherché par la loi ${ }^{\circ}$ 99-05 du 04/04/1999, plusieurs fois amendée, et qui forge l'identité du système actuel. Celui-ci compte désormais soixante établissements d'enseignement supérieur de différents statuts administratifs, dont vingt-sept universités, seize centres universitaires, deux annexes universitaires, six instituts nationaux, cinq écoles nationales et quatre écoles normales supérieures. La diversité est encore réelle. L'ensemble, réparti à travers quarante et une villes universitaires, regroupe 217 structures de prise en charge des activités pédagogiques et 640 laboratoires de recherche scientifiques. À la rentrée universitaire de septembre 2007, l'ensemble de ces établissements a enregistré l'inscription de 1050000 étudiants dont 278000 nouveaux.

L'année 2004/2005 a vu le lancement d'un nouveau régime d'études universitaires portant la dénomination LMD, licence-master-doctorat, en référence au cursus mis en place en Europe dans le cadre du processus de Bologne. Cette réforme, dont la mise en œuvre se fait graduellement en fonction des conditions locales, a été pensée comme une nécessité dictée par l'objectif d'adaptation du système algérien d'enseignement supérieur à l'environnement international, notamment en offrant aux étudiants des formations mieux adaptées à leurs capacités, organisées selon des rythmes plus flexibles. Parmi les autres objectifs, on peut citer la mise en adéquation du diplôme de l'enseignement supérieur avec la demande économique et sociale en matière de compétences, en encourageant le secteur économique public et privé à s'intéresser à la prise en charge des missions de l'enseignement supérieur.

\section{LA PLACE DES SCIENCES SOCIALES DANS LE SYSTÈME D'ENSEIGNEMENT SUPÉRIEUR}

Le second point de notre analyse concerne la place qu'occupent les sciences sociales et humaines dans ce système. Nous nous appuierons principa- 
lement sur l'exemple des sciences économiques, des sciences de l'éducation, de la psychologie et de la sociologie. Quatre moments forts caractérisent l'évolution de leur enseignement.

De la période coloniale à 1972, la formation dans ce qui aujourd'hui est dénommé «sciences humaines et sociales» concernait essentiellement un petit noyau de disciplines - sciences économiques et droit, philosophie, histoire, lettres, psychologie, sociologie ${ }^{3}$ - dont l'enseignement était assuré par deux facultés : celle des lettres et sciences humaines et celle de droit et sciences économiques. Elle débouchait sur une licence "ès sciences économiques» ou «ès lettres»... en sociologie, psychologie, histoire, philosophie, lettres. Ces licences à plusieurs certificats étaient dépourvues d'options. La formation en sciences économiques n'était pas totalement autonomisée par rapport à l'enseignement du droit : licence en droit et licence en sciences économiques partageaient le même tronc commun des deux premières années et ce jusqu'en 1970. Celles en psychologie et sociologie partageaient un tronc commun d'une année dénommé propédeutique.

Cet enseignement des sciences sociales était dispensé dans un seul établissement, l'université d'Alger avec ses deux annexes d'Oran et de Constantine, celles-ci ne prenant en charge que les deux premières années de la licence. De jeunes enseignants algériens formés dans les universités françaises pour la plupart et des enseignants français "coopérants» assuraient l'encadrement scientifique et pédagogique. La recherche et la formation à la recherche étaient assurées par la formation doctorale.

Le second moment fort, de 1972 à 1984, est marqué par la réforme de l'enseignement supérieur, l'introduction de la langue nationale dans toutes les disciplines, et l'arabisation totale de certains enseignements des sciences sociales - dans un premier stade, la philosophie et l'histoire.

Le terme de sciences sociales apparaît dans la nomenclature universitaire à la faveur de cette réforme qui reconfigure la formation selon deux axes complémentaires : autonomisation des disciplines (droit, économie, sociologie, psychologie, philosophie, histoire, archéologie, langues et lettres) et diversification par l'introduction d'un système optionnel.

Le système des facultés fut, comme on l'a vu, supprimé et on réunit les différentes disciplines, désormais enseignées au niveau de départements, dans le cadre d'Instituts. L'université d'Alger, par exemple, spécialisée dans les sciences sociales et humaines, fut restructurée en quatorze instituts: instituts d'études juridiques et administratives (droit), d'économie, des langues étrangères, de langue et de littérature arabes, de sociologie, de psychologie et des sciences de l'éducation, d'histoire, de philosophie, de sciences politiques et des relations internationales, d'information et de communication, d'archéologie, d'éducation physique et sportive, de bibliothéconomie, de traduction et interprétation.

3. La sociologie et la psychologie apparaissent à partir de 1958. 
La licence se déroulait en système modulaire semestriel comptant une bonne cinquantaine de modules répartis en huit semestres, les quatre premiers jouant le rôle de tronc commun, les quatre derniers étant des modules de spécialisation. Pour clôturer la formation, un mémoire de licence était soutenu devant jury. Les sciences économiques, par exemple, comportaient, à partir de la troisième année, sept spécialisations: théorie économique, économétrie, planification, économie financière, gestion, sciences financières, sciences commerciales. La première option à projection fondamentale était conçue pour prendre en charge les meilleurs étudiants et les préparer à assurer la relève en matière d'encadrement scientifique. Les autres options étaient conçues comme des formations appliquées très spécialisées, devant répondre aux différents besoins des entreprises et des administrations centrales et locales.

L'enseignement de la sociologie se diversifiait en quatre spécialités : sociologie générale et politique, industrielle, culturelle, urbaine et rurale. L'enseignement de la psychologie proposait quand à lui deux spécialisations classiques - psychologie clinique et psychologie du travail. On introduisit la nouvelle spécialité «sciences de l'éducation», on institua des formations comme l'orientation scolaire et professionnelle ainsi que l'orthophonie. Quelques années plus tard, on créa des formations techniciennes de courte durée à projection professionnelle (DEUA) répondant plus ou moins à des besoins réels du marché du travail. Ce type de formation constituait pour l'époque une nouveauté pour l'enseignement des sciences sociales et humaines. En psychologie par exemple, une formation en trois ans de techniciens de l'orientation scolaire et professionnelle est proposée. En économie, on propose des formations courtes en informatique de gestion, en statistique appliquée aux sciences sociales, etc.

La formation post-graduée était assurée en partie en Algérie, et en partie à l'étranger, en Europe occidentale - en France en particulier -, aux ÉtatsUnis et Canada, dans les pays d'Europe de l'est et dans les pays du Moyen Orient, notamment l'Égypte. Durant cette période, l'essentiel de l'encadrement scientifique et pédagogique resta assuré par des enseignants coopérants. En sciences économiques, ils étaient majoritairement français. Le français était encore assez largement la langue d'enseignement, mais l'arabisation de l'enseignement en sciences sociales et humaines était en marche.

Le troisième moment fort correspond à la décennie 1982-1992 et se caractérise par trois éléments fondamentaux:

- l'algérianisation de l'encadrement scientifique et pédagogique;

- l'arabisation totale des sciences humaines et sociales;

- l'expansion du système de formation, avec l'apparition d'une multitude de points de formation en sciences humaines et sociales et la croissance des effectifs. 
L'effet le plus important de cette triple tendance réside sans aucun doute dans l'incapacité d'assurer un encadrement suffisant, quantitativement et qualitativement, de la formation supérieure. Si l'algérianisation de l'encadrement scientifique et pédagogique en sciences économiques a permis à beaucoup de jeunes boursiers formés à l'étranger de s'intégrer dans les universités, elle a également introduit beaucoup d'hétérogénéité dans l'encadrement, due notamment à la diversité des lieux de formation, des langues et des cultures, des traditions théoriques et des cadres idéologiques et doctrinaux dans lesquels ont évolué les étudiants algériens formés à l'étranger.

La généralisation de l'arabisation des enseignements en sciences sociales et humaines, à partir de la rentrée universitaire de septembre 1980, a eu pour effet de «démobiliser» l'encadrement initial, et de promouvoir de jeunes enseignants sans expérience de la pédagogie ni maîtrise scientifique achevée des disciplines qu'ils enseignaient.

Un troisième élément est venu compliquer la difficile recherche des équilibres nécessaires à l'émergence d'une véritable école de pensée : l'expansion et la diversification des lieux de formation. Au noyau initial constitué par les universités d'Alger, Constantine et Oran se sont ajoutés plus d'une dizaine d'universités et centres universitaires assurant des formations en sciences sociales et humaines. Cette expansion a contribué à renforcer la contrainte d'encadrement sur le double plan quantitatif et qualitatif.

Cette période a connu également une restructuration des études doctorales. Reposant sur un système à deux paliers constitués par un magister de deux années (se substituant formellement à l'ancien doctorat de troisième cycle) et un doctorat d'État. Chroniquement sous-encadrée, la formation doctorale en sciences sociales a enregistré un faible rendement ne permettant pas d'assurer la reproduction de l'encadrement magistral ou non-magistral. La formation doctorale à l'étranger n'a pas également connu une grande efficacité : très peu de soutenances et surtout très peu de retours. Concomitamment, la recherche scientifique n'a pas connu également une grande efficacité productive, faute non pas tant d'insuffisance de moyens matériels, mais plutôt d'inexistence de structures d'accueil opérationnelles (absence de laboratoires, d'unités et de centres de recherche), d'encadrement de qualité en quantité suffisante et de programmes mobilisateurs. À partir de 1991, une réflexion portant sur l'évolution des programmes de formation est lancée dans un contexte de libéralisation économique et de passage à l'économie de marché, aboutissant en 1995-1996 à une modification importante sous l'effet d'une nouvelle demande en provenance du public estudiantin. Par exemple, en sciences économiques, très peu d'étudiants choisissaient les options «théorie», "planification», «économétrie », "économie financière», ne trouvant plus d'opportunités d'emploi. La masse des étudiants se retrouvait dans deux options : gestion et sciences financières, pour lesquelles des opportunités d'embauche pouvaient exister. 
Il fut décidé de recentrer la licence en sciences économiques sur trois options et d'autonomiser les sciences de gestion et les sciences commerciales en créant des formations propres, avec des programmes spécifiques. En psychologie, la formation en orientation scolaire et professionnelle est remplacée par celle de psychologie scolaire, plus en phase avec les exigences de terrain de réduction des déperditions scolaires et de qualité de l'éducation.

\section{LE POIDS ÉCRASANT DES SCIENCES HUMAINES ET SOCIALES}

Aujourd'hui, l'offre de formations en sciences sociales et humaines est présente dans tous les établissements universitaires. L'examen de l'organisation de cette offre de formation révèle certaines caractéristiques :

- d'abord une faible diversification, avec souvent, dans les différentes filières, une prédominance d'une option sur toutes les autres, drainant une part importante des effectifs des étudiants et provoquant des engorgements peu gérables ;

- une tendance à l'essaimage national par effet de clonage d'une filière sur l'ensemble des établissements, sans que rien ne distingue l'offre de formation d'un site à un autre, où l'on constate toujours la congestion des effectifs et l'insuffisance de l'encadrement;

- la faiblesse, voire l'absence de lien entre l'offre de formation en SHS avec les spécificités des territoires d'implantation des établissements, d'où de lourds problèmes de débouchés professionnels.

Au total, après quarante ans d'évolution, le système algérien de formation universitaire apparaît dominé quantitativement par les formations en sciences sociales et humaines. Cette observation peut être vérifiée par l'examen de l'évolution des effectifs étudiants inscrits dans les établissements d'enseignement supérieur. Depuis les premières réformes universitaires, le nombre d'étudiants en sciences humaines et sociales n'a cessé d'augmenter de façon régulière et quasi concomitante avec le rythme croissant des nouveaux bacheliers. Sur la décennie 1996-2006, le nombre d'inscrits a plus que quadruplé, passant de 120426 à 532953 étudiants. Le taux de croissance annuel moyen des inscrits dans une filière de sciences sociales et humaine avoisine les $15 \%$. Durant certaines années, le taux de croissance des effectifs est extrêmement fort : 29,24\% en 1997/1998, plus de $21 \%$ en 2000/2001 et 2001/2002. S'il est vrai que la tendance à la croissance des effectifs étudiants est générale, le rythme caractérisant les filières de sciences sociales et humaines est beaucoup plus rapide que celui caractérisant celles de sciences «exactes» et technologiques. De ce fait, le poids relatif de l'effectif étudiants poursuivant des études supérieures en SHS augmente très rapidement. Il passe de $42 \%$ de l'effectif total en 
$1996 / 1997$ à près de $53 \%$ en $1999 / 2000$, et à près de $65 \%$ en $2006 / 2007$, représentant près des deux tiers de l'effectif étudiant global.

Cette prédominance des SHS sur toutes les autres disciplines traduit une sorte de spécialisation par défaut de l'université algérienne en SHS de 1999 jusqu'en 2007. On est loin des objectifs de la réforme de 1971, à savoir construire un système universitaire orienté vers la formation technique et technologique afin de répondre aux besoins du schéma d'industrialisation accélérée des années 1970/1980.

Cette tendance marquée à la surcroissance des effectifs étudiants en SHS est à rapporter non seulement au taux de réussite global au baccalauréat, mais surtout au type d'orientation vers les filières universitaires mis en place dès les années 1990. Strictement informatisé, ce système d'orientation, qui essaie en théorie de tenir compte des vœux des candidats, se base d'abord sur la moyenne générale obtenue au baccalauréat, mais surtout à la note précise obtenue dans la discipline majeure de la spécialité, les dix vœux d'orientation exprimés par les étudiants devant se conformer strictement à ces critères de notes. Ces critères draconiens d'orientation des étudiants ferment les portes des formations disciplinaires scientifiques à tout étudiant ayant au-dessous de la note requise, note dont le seuil minimum augmente d'année en année, instaurant une véritable sélection avant l'entrée à l'université. De sorte que l'orientation des bacheliers vers les sciences sociales et humaines est fréquemment une orientation par défaut.

Le plus grand nombre d'étudiants se voient ainsi «relégués", à leur grand désespoir le plus souvent, aux formations en sciences sociales et humaines. Ceci n'a pas manqué de favoriser l'émergence de stratégies développées par les étudiants obligés de «choisir» une formation en sciences sociales et humaines, en faveur des formations considérées comme les plus à même de leur assurer un emploi à leur sortie de l'université. Ainsi deux disciplines se

Ventilation par ordre d'importance des effectifs étudiants inscrits dans une filière de sciences sociales et humaines en 2006/2007

\begin{tabular}{|l|c|c|}
\hline Spécialités & $\begin{array}{c}\text { Nombre d'étudiants } \\
\text { inscrits }\end{array}$ & Taux d'inscription \\
\hline $\begin{array}{l}\text { Sciences économiques/sciences de gestion/ } \\
\text { sciences commerciales }\end{array}$ & 163797 & $30,73 \%$ \\
\hline Sciences juridiques & 105962 & $19,88 \%$ \\
\hline $\begin{array}{l}\text { Sciences sociales (sociologie, psychologie, } \\
\text { sciences de l'éducation, histoire, archéologie) }\end{array}$ & 95402 & $17,90 \%$ \\
\hline Langues et lettres & 68956 & $12,93 \%$ \\
\hline Langues étrangères & 66992 & $12,56 \%$ \\
\hline Sciences politiques et sciences de l'information & 31844 & $5,97 \%$ \\
\hline Total des étudiants en sciences humaines et sociales & $\mathbf{5 3 2 9 5 3}$ & $\mathbf{1 0 0 \%}$ \\
\hline
\end{tabular}

Source : Annuaire statistique du ministère de l'enseignement supérieur et de la recherche scientifique. 
partagent d'une manière inégale près de $51 \%$ des effectifs étudiants inscrits en sciences sociales et humaines : les sciences économiques, sciences de gestion et sciences commerciales, les plus demandées, attirent plus de $30 \%$ du total; tandis que les sciences juridiques captent un peu moins de $20 \%$.

\section{QUEL AVENIR POUR LES SCIENCES HUMAINES ET SOCIALES EN ALgérie?}

Durant près d'un demi-siècle, l'Algérie a consenti un effort considérable pour construire un système d'enseignement supérieur d'une densité suffisante pour couvrir l'ensemble du territoire national. Le nombre d'étudiants va continuer de croître : les projections annoncent des effectifs inscrits d'un million et demi en 2015, et très probablement deux millions et demi en 2025. Ce système a connu jusqu'à maintenant un processus de croissance de type extensif. Les universités algériennes, de création récente, ont connu d'une manière récurrente des périodes très tendues de croissance démographique soutenue, qu'elles ont su canaliser tant bien que mal mais qui les a contraintes à régler dans l'urgence des problèmes quantitatifs au détriment de la recherche de plus de qualité. Les défis qu'elles doivent affronter sont nombreux et complexes : continuer à répondre davantage au principe de proximité sur toute l'étendue d'un territoire considérable, continuer à assurer une place pédagogique à des cohortes d'étudiants de plus en plus nombreuses, améliorer le niveau d'encadrement scientifique et pédagogique, rechercher et trouver les adéquations nécessaires avec le marché du travail, investir de nouveaux champs disciplinaires et rénover les programmes de formation, fournir le cadre nécessaire à l'augmentation de la production scientifique (réseaux et programmes mobilisateurs), s'ouvrir davantage sur le reste du monde, etc.

L'avenir des sciences humaines et sociales ne peut s'envisager hors du cadre du passage au régime d'études universitaires LMD, licence-masterdoctorat. On souhaite aujourd'hui offrir aux étudiants des formations mieux adaptées à leurs capacités, organisées selon des rythmes plus flexibles et débouchant sur la mise en adéquation du diplôme de l'enseignement supérieur avec la demande économique et sociale en matière de compétences. Ceci va-t-il donner une meilleure identité et une meilleure intégration professionnelles aux titulaires d'une licence ou d'un master professionnel en psychologie, en sciences de l'éducation ou en sociologie?

Le nombre très restreint d'inscrits en première année de LMD en sciences sociales à l'université d'Alger en 2007-2008 est-il conjoncturel ou dessine-t-il une tendance nouvelle? Il faudra attendre la généralisation du système LMD, voir l'évolution des effectifs des étudiants qui s'inscriront en sciences sociales et humaines, mais surtout en sciences sociales, pour avoir une véritable image du futur de la formation en sciences humaines et sociales en Algérie. 


\section{BibLIOgRAPHIE}

Annuaire statistique du ministère de l'enseignement supérieur et de la recherche scientifique.

BELARBI Y. et ZIOUR H. (2005) : "Les défis internes de l'enseignement en Algérie», Les Cahiers du Cread, $\mathrm{n}^{\circ} 72$.

BENGUERNA M., LAMRIA A. (2007): "Sociologie en Algérie : état des lieux», Sociologies Pratiques, 137-148.

BOUZID N. (2002): «L'interface enseignement supérieur - monde du travail en Algérie : de quoi s'agit-il ?» Les Cahiers du Cread, nº 59-60, 185-201.

FEROUKHI D., FRAIHAT HAKMIA S. (2001): "À la quête du premier emploi : jeunes diplômés en licences d'enseignement et en D.E.S.», Les Cahiers du Cread, $\mathrm{n}^{\circ} 58,97-120$.

FERROUKHI D. (1991): «Insertion professionnelle des diplômés de l'Institut des sciences économiques d'Alger», Les Cahiers du Cread, nº 26, 97-120.

GHALAMALLAH M. (2003): "Attitudes et pratiques professionnelles des enseignants universitaires algériens", Les Cahiers du Cread, $\mathrm{n}^{\circ}$ 62-63.

GHALLAMALLAH M. (2006): «L'université algérienne: genèse des contraintes structurelles, conditions pour une mise à niveau », Les Cahiers du Cread, $\mathrm{n}^{\circ}$ 77, 31- 52.

KADRI A. (2002) : «Pédagogies et société : au fondement de l'anomie du système de l'enseignement supérieur algérien", Les Cahiers du Cread, n 59-60. 2002.

LAMARA G. (2006): "La recherche universitaire en Algérie: des équipes de recherches aux laboratoires période 1962-2003 : particularités d'un cheminement", Les Cahiers du Cread, $\mathrm{n}^{\circ}$ 77, 129-143.

MEKIDECHE T. (1996) : «Connaître et reconnaître la psychologie scolaire en Algérie », Annales de l'Université d'Alger, numéro spécial (Le système éducatif algérien: les enjeux du changement), Alger, 94-107. 
